https://helda.helsinki.fi

\title{
Thermal barriers constrain microbial elevational range size via climate variability
}

\section{Wang, Jianjun}

2017

Wang , J \& Soininen , J 2017 , ' Thermal barriers constrain microbial elevational range size via climate variability ' , Environmental Microbiology , vol. 19 , no. 8 , pp. 3283-3296 . https://doi.org/10.1111/1462-29

http://hdl.handle.net/10138/217120

https://doi.org/10.1111/1462-2920.13823

Downloaded from Helda, University of Helsinki institutional repository.

This is an electronic reprint of the original article.

This reprint may differ from the original in pagination and typographic detail.

Please cite the original version. 


\section{Thermal barriers constrain microbial elevational range size via climate variability}

\author{
Jianjun Wang (D)1,2*† and Janne Soininen ${ }^{2 \dagger}$ \\ ${ }^{1}$ State Key Laboratory of Lake Science and \\ Environment, Nanjing Institute of Geography and \\ Limnology, Chinese Academy of Sciences, Nanjing \\ 210008, China. \\ ${ }^{2}$ Department of Geosciences and Geography, University \\ of Helsinki, Helsinki FIN-00014, Finland.
}

\section{Summary}

Range size is invariably limited and understanding range size variation is an important objective in ecology. However, microbial range size across geographical gradients remains understudied, especially on mountainsides. Here, the patterns of range size of stream microbes (i.e., bacteria and diatoms) and macroorganisms (i.e., macroinvertebrates) along elevational gradients in Asia and Europe were examined. In bacteria, elevational range size showed nonsignificant phylogenetic signals. In all taxa, there was a positive relationship between niche breadth and species elevational range size, driven by local environmental and climatic variables. No taxa followed the elevational Rapoport's rule. Climate variability explained the most variation in microbial mean elevational range size, whereas local environmental variables were more important for macroinvertebrates. Seasonal and annual climate variation showed negative effects, while daily climate variation had positive effects on community mean elevational range size for all taxa. The negative correlation between range size and species richness suggests that understanding the drivers of range is key for revealing the processes underlying diversity. The results advance the understanding of microbial species thermal barriers by revealing the importance of seasonal and diurnal climate variation, and highlight that aquatic and terrestrial biota may differ in their response to short- and long-term climate variability.

Received 5 December, 2016; accepted 7 June, 2017. *For correspondence. E-mail jjwang@niglas.ac.cn; Tel. +86-25-8688-2219; Fax $+86-25-5771-4759 .{ }^{\dagger}$ These authors contributed equally to this work.

\section{Introduction}

Range size, the 'fundamental unit' of macroecology, is generally geographically limited, and the study of how and why species range size is constrained is a central objective of ecology (Gaston and Blackburn, 2000; Gaston, 2003). The systematic variation in range size along geographical gradients (i.e., elevation or latitude) represents an important underlying driver of species richness patterns (Stevens, 1992; Brown et al., 1996; Colwell and Lees, 2000). This range size variation and the corresponding biodiversity patterns are affected by climate changes and human activities: small-ranged species in particular are more vulnerable to large-scale environmental changes (Thuiller et al., 2005; Chen et al., 2011; McCain and Colwell, 2011; Morueta-Holme et al., 2013; Krosby et al., 2015; Mellin et al., 2016). A deeper understanding of the ecological determinants of species range size is critical to predicting organism responses to climate change and human activities (McCain and Colwell, 2011).

Climate and climate variability impose selective pressures on the elevational ranges of terrestrial vertebrates (McCain, 2009; Sheldon et al., 2015; Chan et al., 2016), indicating that local environmental factors are less important than climatic variables for determining elevational range size. Compared with marine and terrestrial species, freshwater species represent the most vulnerable organisms to climate change (Wiens, 2016), yet, their range size across geographical gradients remains less explored, especially along elevational gradients. Furthermore, although spatial community patterns have recently been documented more extensively for microbes (Martiny et al., 2006; Fierer et al., 2009; Hanson et al., 2012), range size and the underlying drivers across typical climatic gradients (e.g., elevations or latitudes) are still understudied, although a few studies have carried out (e.g., Wang et al., 2011; Azovsky and Mazei, 2013; Miyamoto et al., 2014). This is especially true in mountainous regions with strong climatic gradients, which could potentially represent the ideal settings for exploring fundamental questions regarding range size. For instance, are there phylogenetic signals in species range size for microbes? How do different categories of environmental variables, such as local abiotic factors, climate and climatic variation, affect microbial 
species distributions and their underlying range size? To what extent does climate affect species range size with respect to other factors?

Range size studies typically employ a broad scope of methods to characterize species range limits. Species elevational range size can be plotted against their elevational midpoints, which can in turn be used to explain the humpshaped elevational patterns of species richness with middomain hypotheses (Colwell and Lees, 2000). Moreover, niche breadth, defined as the range of environmental conditions and resources that a species utilizes (MacArthur, 1968), may be a useful predictor of species range size because traits in general are known to be related to environmental and geographical gradients in microbes (Soininen et al., 2016), and niche breadth has been documented to correlate well with range size (Gaston and Blackburn, 2000; Slatyer et al., 2013; Trakimas et al., 2016). In addition, meta-analyses have shown a positive relationship between range size and niche breadth, classified as environmental tolerance breadth, habitat breadth and diet breadth (Slatyer et al., 2013). The niche breadthrange size hypothesis (NBRS) is an important focus of recent ecological studies because it proposes mechanisms for commonness and rarity, and it is used to predict species' vulnerability to extinction under rapid global change (Slatyer et al., 2013). Similar to what has been found in plants and animals (Slatyer et al., 2013; Papacostas and Freestone, 2016), we expect a positive correlation between niche breadth and geographical range size in aquatic microbial taxa on mountainsides. We further expect that climate and climate variability may have stronger effects than local environmental variables on species elevational ranges (Chan et al., 2016).

Species elevational range size has been theorized to increase toward higher elevations; this prediction has been known as the elevational Rapoport's rule (ERR) (Stevens, 1992) and represents an extension of the latitudinal Rapoport's rule (Stevens, 1989), which suggested that climate variability may be the underlying mechanism for range size (Stevens, 1989; 1992; Chan et al., 2016). The ERR has also been invoked to explain species elevational distributions and species richness patterns (Stevens, 1992). The ERR follows Janzen (1967) in postulating that because climatic conditions typically vary more at higher elevations, species occurring at high elevations must have broader climatic tolerances (i.e., broader environmental niches), and hence, larger elevational range size than species occurring at low elevations. Climatic tolerances are traits typically shaped by evolutionary selection and manifested in the realized elevational and geographical ranges of species (Janzen, 1967; Eckert et al., 2008). However, many higher taxa such as plants or terrestrial vertebrates do not seem to comply with the ERR (e.g., McCain and Bracy, 2013). Most importantly, tests of this rule in microbes and underlying mechanisms for species range size are still rare or completely missing (but see Teittinen et al., 2016). Species range size is the product of multiple determinants such as taxonomic group, ecosystem type, latitude, trophic group, abundance, body size, species interactions and dispersal ability (Brown et al., 1996; Gaston, 2003), and are considered a predictor of the vulnerability of a species to climate change and other disturbances (Angert et al., 2011). Thus, species range size patterns and associated climatic niche breadths are of paramount importance for understanding species responses to rapid environmental change.

Here, we investigated patterns of aquatic microbial range size at both species and community levels along elevational gradients in streams, and compared the findings with the corresponding elevational biodiversity data for benthic macroinvertebrates sampled at the same sites. We employed regressions and meta-analyses to assess the main drivers of range size using climatic and local environmental variables and species traits (i.e., niche breadth). We had three hypotheses regarding species elevational range size:

(1) Microbes will not show significant phylogenetic signals in species elevational range size because significant phylogenetic signals are rarely found in plants and animals (Gaston, 2003).

(2) Species elevational range size will correlate positively with niche breadth. This is because a species would become more widespread by utilising a greater array of resources and maintaining a nonnegative population growth rate across a wider range of environmental conditions (Hanski, 1982; Brown, 1984; Slatyer et al., 2013).

(3) Species elevational range size will show strong relationships with climatic drivers. In addition, climatic variables, especially climate variability, are expected to be a stronger determinant of range size than local environmental variables, especially when large climatic gradients are considered (Chan et al., 2016; Pajunen et al., 2016). We expected the short- and long-term climate variability will also have opposite effects on elevational range size for aquatic biota similarly as found for terrestrial vertebrates (Chan et al., 2016).

\section{Results}

Meta-analysis of species elevational range size

Mantel correlograms consistently showed that nonsignificant $(P>0.05)$, positive correlations across phylogenetic distances were dominant for the bacterial species elevational range size in the six elevational gradients (Fig. 1). Significant phylogenetic signals were observed in some 


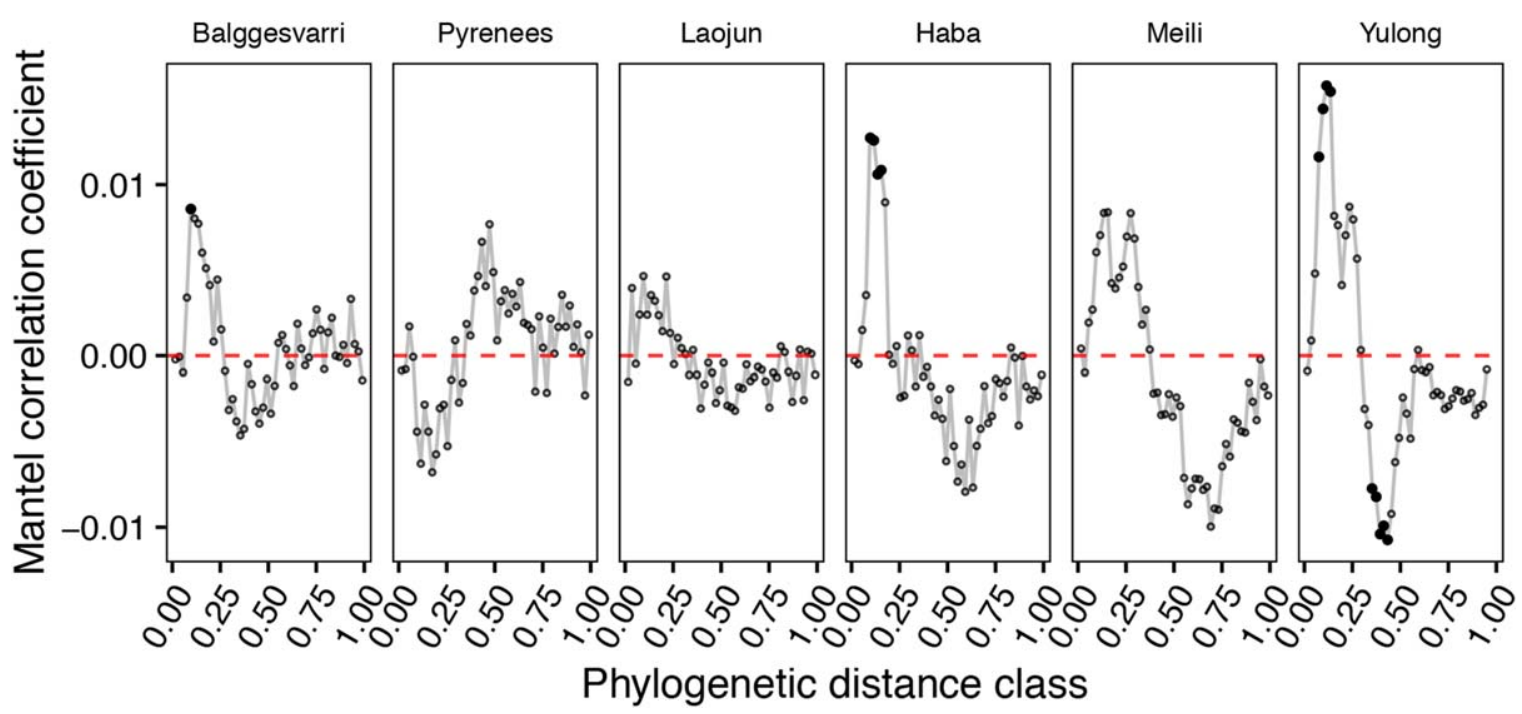

Fig. 1. Pearson correlation resulting from Mantel correlogram between the pairwise matrix of bacterial species elevation range size differences and phylogenetic distances for each mountain, with 999 permutations. Phylogenetic distance was standardized to range from 0 to 1.

Significant correlations $(P \leq 0.05$, solid circles) indicate phylogenetic signal in species range size, and were rarely found across phylogenetic distances for any mountainside.

cases only at the intermediate, but not at the short phylogenetic distances (Fig. 1).

For the mean annual temperature (MAT) niche breadth, NBRS was significant $(P<0.05)$ in almost all gradients across the three taxonomic groups (Supporting Information Fig. S1). The niche breadth of the five environmental categories correlated significantly $(P<0.05)$ with species elevational range size across streams and taxonomic groups, especially in bacteria (Supporting Information Fig. S2).

There were significant $(P<0.05)$, positive mean effect size of NBRS for each niche category and for each taxonomic group (Table 1). Regarding niche category and taxonomic group, we found the highest mean effect size of NBRS was for MAT (0.463) and diatoms (0.469), while the mean effect size was lowest for climatic variation (0.371) and macroinvertebrates (0.277) (Table 1). Heterogeneity in effects size was high for each niche category among taxonomic groups, with a range of $91.5-97.0 \%$ of the total variation in estimated effect sizes (Table 1). When each taxonomic group was considered separately, the high heterogeneity in effect sizes was observed only for bacteria $(98.40 \%)$, but not for the other two taxa. Niche category (Supporting Information Table S1) and taxonomic group (Supporting Information Table S2) did not have a significant impact on mean effect size, except for the chemistry variables (Supporting Information Table S2).

\section{Mean elevational range size at the community level}

When using the Stevens' method (Stevens, 1992) we found that the mean elevational range size of all species in a community showed variable elevational patterns, ranging from significantly positive to negative relationships among taxonomic groups and elevational gradients (Fig. 2). This result contrasts with the prediction of ERR that the species elevational range size would increase toward higher elevations. Support for ERR was obtained for only two data sets: Meili for bacteria and Laojun Mountains for macroinvertebrates (Fig. 2). Using McCain's method (McCain and Bracy, 2013) we further confirmed that no solid evidence for a consistent ERR was found across mountains (Supporting Information Fig. S3). In general, the patterns in mean range size of bacterial communities were largely similar to those of macroinvertebrates (Fig. 2; Supporting Information Fig. S3). Interestingly, species richness decreased consistently with increasing mean elevational range size of the species in a community across all taxonomic groups and mountains (Supporting Information Fig. S4).

Generally, mean elevational range size was significantly $(P<0.05)$ correlated with the measured environmental variables for the full data set of taxonomic groups (Supporting Information Figs S5-S7). For all taxonomic groups, elevational range size had a significant $(P<0.05)$ positive relationship with MAT and mean diurnal range (MDR), and a negative relationship with temperature seasonality (TS) (Supporting Information Figs S5-S7). However, the above relationships were stronger for bacteria and diatoms than for macroinvertebrates, with higher $R^{2}$ values (Supporting Information Figs S5-S7). For each stream, the linear models with the lowest Akaike's Information Criterion (AIC, Yamaoka et al., 1978) indicated that the factors correlating with mean range size were most frequently MDR, 
Table 1. Effect sizes and confidence intervals for the relationships between niche breadth and species elevation range size, and the test for heterogeneity using the random-effects meta-analysis (RE model).

\begin{tabular}{|c|c|c|c|c|c|c|c|}
\hline \multirow[b]{2}{*}{ Categories } & & \multirow[b]{2}{*}{$N$} & \multirow[b]{2}{*}{ Mean effect $(z)$} & \multirow[b]{2}{*}{$95 \% \mathrm{Cl}$} & \multirow[b]{2}{*}{$P$} & \multicolumn{2}{|l|}{ Heterogeneity } \\
\hline & & & & & & $Q_{\mathrm{T}}(P)$ & p \\
\hline \multirow[t]{5}{*}{ Niche breadth } & Stream morphology & 16 & 0.438 & $0.384,0.492$ & $<0.0001$ & $156.72(P<0.0001)$ & $91.79 \%(76.80,98.54)$ \\
\hline & Chemistry & 16 & 0.391 & $0.310,0.472$ & $<0.0001$ & $309.55(P<0.0001)$ & $96.98 \%(91.36,99.10)$ \\
\hline & Nutrients & 16 & 0.448 & $0.376,0.521$ & $<0.0001$ & $337.39(P<0.0001)$ & $95.97 \%(88.32,98.56)$ \\
\hline & MAT & 16 & 0.463 & $0.395,0.531$ & $<0.0001$ & $278.85(P<0.0001)$ & $95.26 \%(86.33,98.70)$ \\
\hline & Climatic variation & 16 & 0.371 & $0.318,0.424$ & $<0.0001$ & $337.39(P<0.0001)$ & $91.49 \%(75.56,98.29)$ \\
\hline \multirow[t]{3}{*}{ Taxonomic group } & Bacteria & 30 & 0.440 & $0.402,0.477$ & $<0.0001$ & $1535.82(P<0.0001)$ & $98.40 \%(97.47,99.12)$ \\
\hline & Diatoms & 30 & 0.469 & $0.410,0.527$ & $<0.0001$ & $29.29(P=0.450)$ & $4.77 \%(0.00,44.37)$ \\
\hline & Macroinvertebrates & 20 & 0.277 & $0.201,0.352$ & $<0.0001$ & $14.743(P=0.739)$ & $0.00 \%(0.00,38.06)$ \\
\hline
\end{tabular}

Stream morphology: stream width, shading, substratum size, depth and current velocity. Chemistry: pH and conductivity. Nutrients: TP (total phosphorus) and CDOM (chromophoric dissolved organic matter). MAT: mean annual temperature. Climatic variation: MDR (mean diurnal range), TS (temperature seasonality) and TAR (temperature annual range).

temperature annual range (TAR) and conductivity for bacteria, TS, TAR, width and total phosphorus (TP) for diatoms, and current velocity for macroinvertebrates (Fig. 3).

Based on the boosted regression trees (BRT, Elith et al., 2008) results obtained for the six streams, TS was the most important variable for explaining mean range size in bacteria and diatoms, followed by MDR (Fig. 4A and B;
Supporting Information Figs S8 and S9). However, chromophoric dissolved organic matter (cDOM) was the most important variable for explaining mean range size in macroinvertebrates, followed by MDR, TS and riparian shading (Fig. 4C; Supporting Information Fig. S10). For the Chinese streams, temperature-related factors were still the most important variables for explaining mean range size in

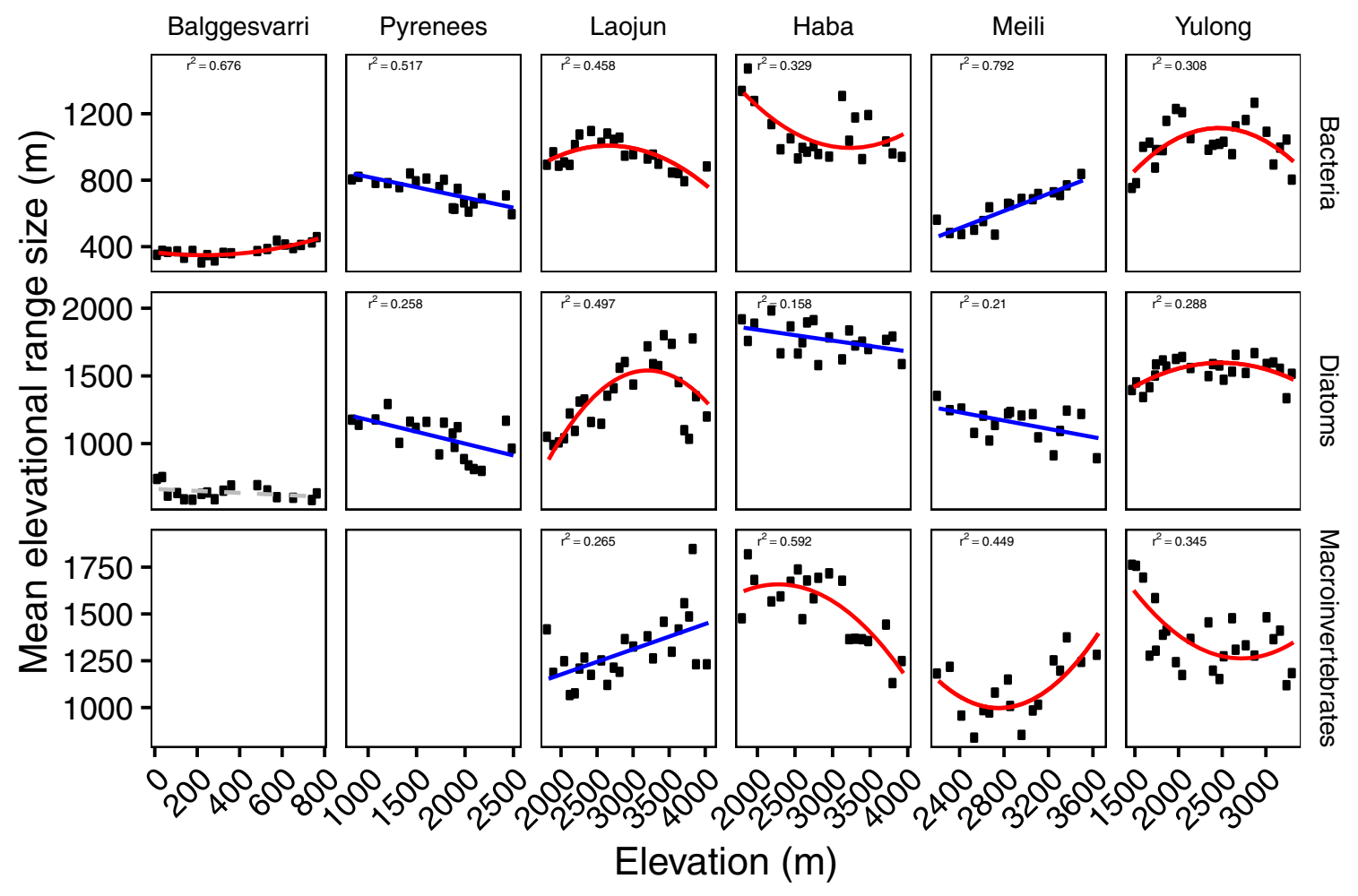

Fig. 2. The relationship between community mean elevational range size and elevation. Mean elevational range size is the mean value of species elevation range for all species in the community based on Stevens' method. The relationship for each elevation band was fitted by linear and quadratic models, the significance of which $(P \leq 0.05, F$-test) is shown with blue and red lines. The better model, shown with a solid line, was selected based on lower value of Akaike's information criterion. Adjusted $R^{2}$ values are shown for significant $(P \leq 0.05, F$-test) linear (blue) and quadratic (red) regressions. A non-significant $(P>0.05, F$-test) relationship is shown by a grey dotted line. [Colour figure can be viewed at wileyonlinelibrary.com] 

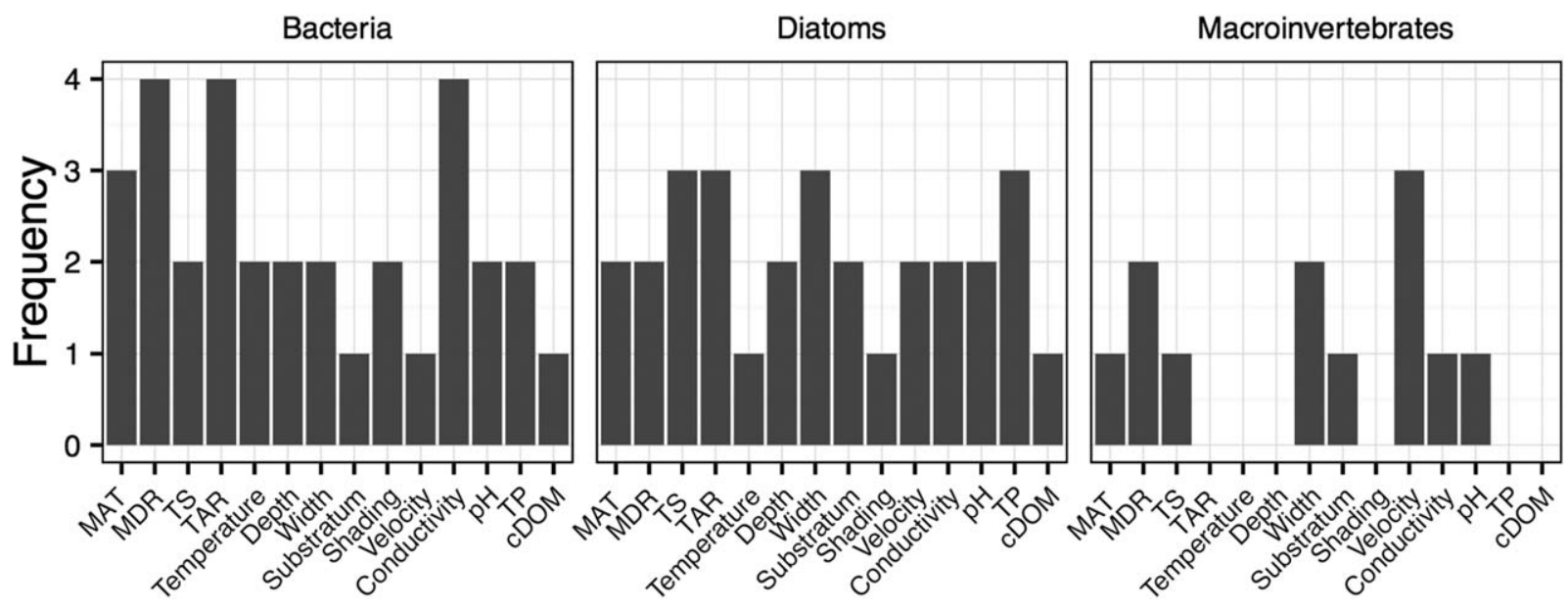

Fig. 3. Environmental explanatory variables for mean elevational range size across streams and taxonomic groups based on a linear model. The best models were identified using Akaike's information criterion. All of the environmental variables were standardized $(m e a n=0 ; S D=1)$. The frequency of the significant $(P<0.05)$ explanatory environmental variables were summarized based on the results of linear models. Temperature: streamwater temperature. TP: total phosphorus. Shading: riparian shading (\%). Substratum: median of the substratum particle size. Velocity: current velocity. Depth: streamwater depth. cDOM: chromophoric dissolved organic matter. Temperature: streamwater temperature. MAT: mean annual temperature. MDR: mean diurnal range. TS: temperature seasonality. TAR: temperature annual range.

bacteria (TS and MDR, Supporting Information Fig. S11A) and diatoms (MDR and streamwater temperature, Supporting Information Fig. S11B).

\section{Discussion}

Although the geographical range of a species is a basic concept in biogeography and macroecology (Brown et al.,
1996; Gaston, 2003), the patterns and drivers of microbial species range size across geographical gradients such as elevations remain understudied. Here, we studied the species elevational range size of bacteria, diatoms and macroinvertebrates from six mountains in three regions in Asia and Europe. Our results highlight five main findings. First, phylogeny cannot predict species elevational range in bacteria. Second, there was a consistent and general

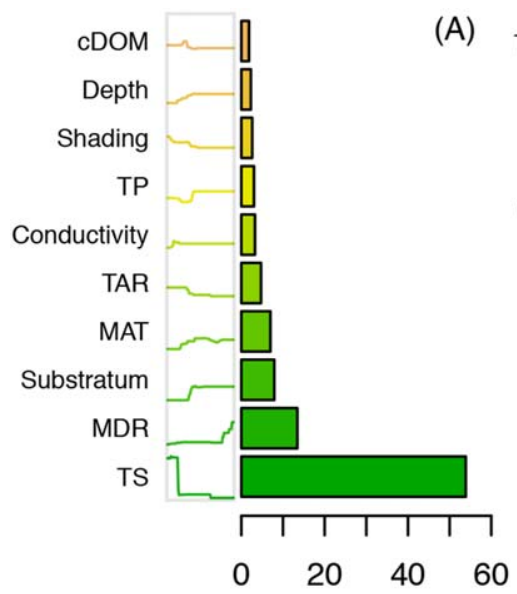

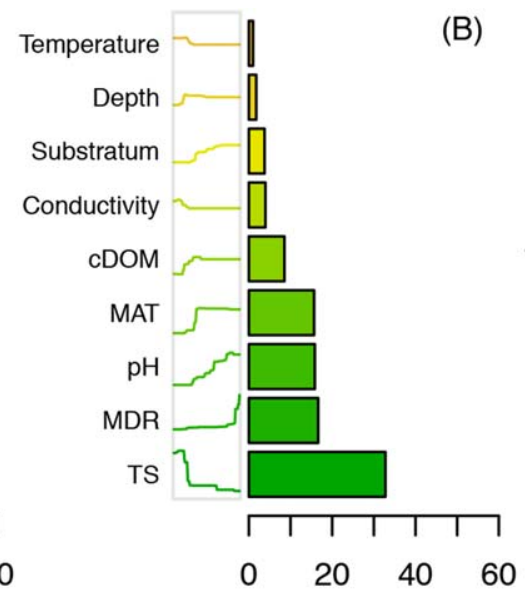

Relative proportion

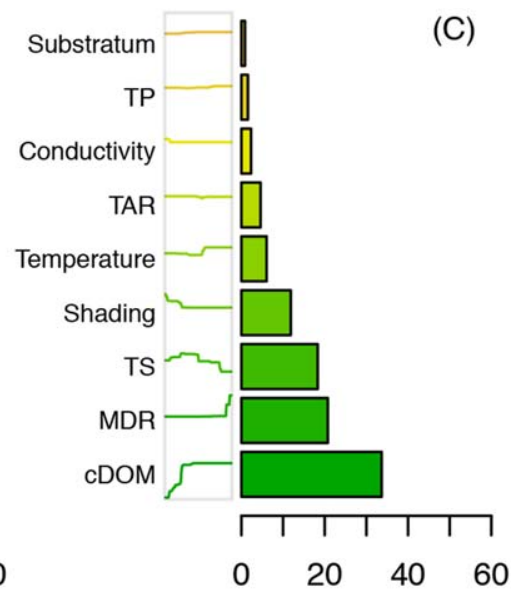

Fig. 4. The environmental factors related to the community elevational range size, identified with Boosted Regression Trees (BRT). The results were for bacteria (A) and diatoms (B) from all six streams and macroinvertebrates (C) from the four streams in China. The values of the relative contribution (\%) of each variable for each biodiversity metric are shown as bar plots. The solid lines at the left side of bars indicate the BRT-modelled response curves to the corresponding variables, where the $y$-axes represent the marginal effect of the respective explanatory variable (centred to zero mean) on the predicted mean elevational range size. For better visualization, the $\mathrm{x}$ - and $\mathrm{y}$-axes of the solid lines were not shown, but detailed information on BRT-modelled response curves can be found in Supporting Information Figs S8, S9 and S11. TP: total phosphorus. Shading: riparian shading (\%). Substratum: median of the substratum particle size. Velocity: current velocity. Depth: streamwater depth. cDOM: chromophoric dissolved organic matter. Temperature: streamwater temperature. MAT: mean annual temperature. MDR: mean diurnal range. TS: temperature seasonality. TAR: temperature annual range. [Colour figure can be viewed at wileyonlinelibrary.com] 
relationship between niche breadth and species elevational range size across all taxonomic groups, and both local environmental and climatic variables contributed strongly to these relationships. Third, we found that no taxon follows the ERR, indicating that species elevational range size did not increase toward higher elevations. Fourth, at the community level, climate variability was the most dominant factors for explaining mean elevational range size in microbes, whereas local environmental variables were more important than climatic variables for explaining range size in macroinvertebrates. Finally, daily climate variation related positively with elevational range size for all taxa, while longer-term climatic variability scaled negative with range size. Below, we discuss these main findings in detail.

For the first time, we showed that significant phylogenetic signal in species elevational range size was rarely observed for the stream bacteria, which indicates that the species elevational range of bacteria exhibit remarkably little phylogenetic conservatism. This phenomenon is consistent with previous studies in plants and animals that found no phylogenetic conservatism in range size, despite considerable systematic variation in geographical range size in major groups of macroorganisms (Brown et al., 1996; Gaston, 2003). Our findings are also consistent with those of a recent study of soil bacteria (Barberán et al., 2014), which showed that evolutionary history cannot explain differences in species occupancy. The decoupling between phylogeny and species range size in bacteria could be the result of the high phylogenetic and metabolic diversity found in bacterial species, the high degree of functional plasticity (Tettelin et al., 2008), and/or the high dispersal ability of species across the studied mountain regions (Wang et al., 2016). Thus, even closely related bacterial species with similar biogeographical and evolutionary histories can vary tremendously in geographical range size. As deterministic processes typically drive microbial community composition among habitats (Wang et al., 2013), and because trait information is missing for most species, we further examined the correspondence between niche breadths and elevational range size.

By considering three different taxa and multiple mountains, we demonstrated that the NBRS relationship is a general ecological pattern across micro- and macroorganisms. This positive NBRS relationship emerged even when we estimated niche breadth and species elevational range size using as independent data sets as possible generated with consistent field sampling and species identification methods. The pattern is consistent with the findings of a recent meta-analysis on NBRS relationships mainly in plants and animals (Slatyer et al., 2013). Our results highlight the possibility of predicting local or regional species elevational range size (e.g., in Pyrenees Mountain) based on the estimated global niche breadth values estimated.
However, there are certain differences in the strength of the NBRS relationships between micro- and macroorganisms. For instance, among the three taxonomic groups, the weakest NBRS relationship was found in macroinvertebrates (average effect size of 0.277 ), whereas the strongest relationship was found for diatoms (average effect size of 0.469 ), which is also quite close to that found in bacteria (Table 1). The effect size of macroinvertebrates is far lower than what has previous been reported for passerine birds $(z=0.43)$ (Laube et al., 2013) or for plants and animals $(z=0.45)$ (Slatyer et al., 2013); however, effect sizes in bacteria and diatoms are relatively similar to what has been reported for larger organisms. Furthermore, the different results for niche breadth of chemical variables among the three taxonomic groups showed that macroinvertebrates had significantly different mean effect sizes for stream chemistry, compared with the other two groups. This might be largely due to the differences in dispersal ability, habitat availabilities, or home range requirements among the various taxonomic groups. For instance, birds have on average larger geographic ranges than do mammals (Gaston, 2003), while microbes have larger elevational range size than macroinvertebrates, perhaps due to dispersal via air and across large distances over continents (Liu et al., 2016; Wang et al., 2016). Thus, for the first time, we have highlighted significant differences in NBRS relationships between micro- and macroorganisms; such comparisons had been missing from previous studies because microorganisms have simply been less considered overall (Gaston, 2003; Slatyer et al., 2013).

When we looked at the five niche axis categories, the strongest NBRS relationship was found for mean annual temperature, suggesting that a higher temperature niche breadth may facilitate mountainside occupancy (Supporting Information Table S1). In contrast, the niche breadth of climatic variation showed the weakest relationship with elevational range size, suggesting that this variable cannot fully explain the elevational range size. Overall, these results not only indicate that the climatic niche breadth is an important predictor of elevational range size but also that the other local environmental niche breadths, such as stream morphology, chemistry and nutrients, may play a role in shaping range size and help predict future of range size under large-scale environmental changes. Nonetheless, we conclude that the significant effects of climatic variables highlight the importance of the climatic hypothesis: organisms that experience a wider range of temperatures or greater temperature variability and thus have broader physiological thermal tolerances, tend also to be geographically widely distributed as a consequence (Spicer and Gaston, 2009; Morueta-Holme et al., 2013; Chan et al., 2016).

We did not find evidence for a general elevational Rapoport's rule (Stevens, 1992) in stream organisms, either with Steven's (Stevens, 1989; 1992) or with McCain's 
methods (McCain and Bracy, 2013). Our findings, however, are consistent with observations made in a wide range of terrestrial taxonomic groups (reviewed by McCain and Bracy, 2013), stream biofilm diatoms (Teittinen et al., 2016) and some fish studies (Fu et al., 2004), suggesting that there is not a consistent positive range size-elevation relationship. However, Beketov (2009) found that, along a 2500-m elevational gradient in Russia, mayfly species elevational ranges expanded with the increase in the ranges' midpoints and that the mean range per elevational band increased with elevation and concurrent decrease in species richness. Furthermore, a similar positive range sizeelevation relationship was documented in Himalayan fish $(0-3800 \mathrm{~m})$ (Bhatt et al., 2012), in Mediterranean beetles $(0-2458 \mathrm{~m})$ (Fattorini, 2014) and in Swiss black scavenger flies (200-2000 m) (Rohner et al., 2015). These contrasting findings suggest that either the outcome depends on specific characteristics of the species under study (climatic tolerance, dispersal ability, overall niche characteristics of species) or on the length or steepness of the environmental gradients prevailing in the study area. Although climatic variation (both seasonal and diurnal) typically increased with elevation according to climatic data in our study, such an increase was probably too subtle to result in consistently larger range size toward higher elevations. Although we compiled the largest data set so far on the elevational range size of aquatic organisms, any general conclusion about ERR is still highly premature for aquatic taxa at present. The contradictory findings call for further studies on elevational range size across a wide range of taxa and ecosystems with different biological and environmental features. We also suggest to go beyond simply describing elevational range size patterns and more actively seek mechanistic explanations behind such patterns (e.g., Janzen, 1967; Chan et al., 2016; Payne and Smith, 2017).

Based on previous studies, we hypothesized that community mean range size in bacteria and diatoms would most likely be constrained by chemical variables such as $\mathrm{pH}$, organic carbon and nutrient concentrations (Soininen, 2007; Wang et al., 2011), whereas range size in macroinvertebrates would be influenced more directly by physical variables such as stream size, current velocity and stone size (Mykrä et al., 2007). In contrast, present results suggest that the ranges of all taxonomic groups seem to be mostly constrained by climatic variables (especially by TS and MDR), although the chemical variable CDOM was the most important variable constraining range size in macroinvertebrates. Most likely, because they act as decomposers, the mean range size of bacteria did not show predictable patterns among nutrients, such as carbon and phosphorus, although bacterial diversity has been reported to correlate with organic carbon and total phosphorus in streams (Wang et al., 2011; 2017). The correlation between macroinvertebrates and organic matter could be explained by their dependency on organic matter (such as leaves). For instance, some stream macroinvertebrates often use leaves for nest building and feeding (such as shredders or and collectors, which feed on coarse- and small-particle organic matter respectively) (e.g., Dudgeon and Wu, 1999). For the three aquatic taxa groups, the pivotal importance of climatic variations on community range size is generally consistent with results from recent studies in terrestrial plants and animals (McCain, 2009; Chan et al., 2016), and we also documented opposite effects of seasonal and daily climate variation in explaining elevational range size of species at focal sites, as found for vertebrates (Chan et al., 2016). These findings are strongly supportive of a temperature mechanism underlying species range size for aquatic organisms across mountains and taxa.

However, contrary to Chan et al.'s (2016) major findings of the influences of climate variability with different temporal scales, that is, seasonal and diurnal, on community elevational range size, we found negative effects of seasonal climate variation on ranges, but positive effects of daily climate variation. For instance, our observation does not support that long-term temperature variation (e.g., annual or seasonal variation) should affect elevational range sizes by constraining ranges where temperature variation is minimal and expanding ranges where temperature variation is large, which is known as Janzen's hypothesis or climate variability hypothesis (Janzen, 1967; Sheldon et al., 2015; Chan et al., 2016). There might be two reasons why different scales in temperature variability have contrasting effects on range size, between our results and those of Chan et al. (2016). (1) Different spatial scales in range size measurements were used. Chan et al. (2016) estimated mean elevational range size among all species for each taxonomic group on each mountain, while we used mean elevational range size of species for each focal assemblages occurring at sampling sites across mountains and taxa. The former metric focuses more on larger, regional spatial scales, and thus might detect positive effects of variations in temperature at coarser scales, such as annual and seasonal. (2) Organisms in different ecosystems and with different life spans were used. Chan et al. (2016) considered both endothermic to ectothermic vertebrates mainly from terrestrial environments, while we focused on bacteria, diatoms, and macroinvertebrates from aquatic environments. These aquatic organisms have smaller body size and shorter life span, are ectothermic, and thus perhaps more sensitive to the variation in ambient temperature, especially at shorter timescales. They are also not limited by water availability, as opposed to terrestrial species which are physiologically affected by precipitation patterns, such as mean annual precipitation for terrestrial vertebrates (Chan et al., 2016). The sensitivity to variation in temperature perhaps explains the positive 
effects on range size of temperature at shorter temporal scales (i.e., diurnal), but the negative effects at longer scales (i.e., seasonal and annual) in stream taxa. It can be envisaged that active thermoregulation of endotherms may enable them to adapt better to diurnal extreme variations in temperature (McCain, 2009), whereas running water in streams enable aquatic organisms to better buffer annual or seasonal extreme variations in temperature compared with terrestrial vertebrates.

Our results also show that species richness and the mean range size of species occurring at focal sites were significantly related, showing a consistent strong negative relationship. This lends support to an idea that understanding the drivers of geographical variation in range size may be key for revealing the processes that underlie species diversity (Stevens, 1989; Graves and Rahbek, 2005), and further suggests that richness and range size either had at least partly similar drivers or that such diversity-range size relationships were driven not only by biological constraints but also by pure mathematics (Arita et al., 2008; Soberón and Ceballos, 2011). In our sample of small aquatic taxa, which comprised many rare species, biological reasons could be put forward to explain our findings. (1) First, species richness values were strongly affected by the distribution of rare species, such that sites with high species richness harboured a higher number of rare species. As locally rare species typically have small range size (Brown, 1984), richness and range size tend to show negative relationships. (2) Second, as range size was correlated with niche breadth, locally rare but numerous species with small niche breadth were able to coexist due to the efficient division of niche space with a small number of generalists (Klopfer and MacArthur, 1960). (3) Third, another reason could be that the relationships among the many measures of spatial patterns of biological diversity, such as $\alpha-, \beta$ - and $\gamma$-diversity or range size, are simply constrained by mathematics because all measures are derived from the same presence-absence or abundance matrix (Soberón and Ceballos, 2011). Such mathematical constraints have recently been addressed through the development of new methods to account for relationships between different biodiversity components (e.g., Kraft et al., 2011; Legendre and De Cáceres, 2013; Legendre, 2014). (4) Finally, range-based stochastic models of species richness gradients (Colwell and Hurtt, 1994; Colwell, 2011) demonstrate that sampling constraints might also result in negative relationships between species richness and mean range size of species at focal sites. Negative relationships between species range size and species richness do not suggest that Rapoport's rule can be generally applied for elevational gradients in explaining species richness, as Steven suggested (Stevens, 1989; 1992). However, the very different elevational patterns in species richness for stream and soil microbes (Wang et al., 2017) reveal a challenge in plugging in universal demographic, ecological or evolutionary theories for observed biodiversity within and across mountains.

Regarding the environmental or climatic variables affecting biological range size along elevational gradients, several concerns need to be taken into account. First, species range size is sensitive to sample size per species, and, in this study, equal-effort sampling of assemblages that vary in underlying species richness might result in spuriously high correlation between richness and range size (Colwell and Hurtt, 1994). One possible solution to this problem is to equalise sample coverage (Chao and Jost, 2012) instead of adjusting sampling effort. We thus estimated species richness and calculated species elevational range size based on the lowest sample coverage for each taxonomic group (Chao and Jost, 2012; Hsieh et al., 2016), and found three reasons to support our choice of effort-based method. (1) Our results confirmed the negative relationships between species richness and mean elevational range size across mountains and taxa, no matter whether the species richness was effort-based (Supporting Information Fig. S4) or coverage-based (Supporting Information Fig. S12). In contrast, however, coverage-based method (Supporting Information Fig. S12) mostly showed higher correlations between species richness and range size than effort-based method for all taxonomic groups (Supporting Information Fig. S4). (2) Interestingly, we further found high correlations between effort-based and coverage-based species richness (Supporting Information Fig. S13) and the correlation was extremely high when we used the rarefied species $X$ site matrix (that is, with the same sequencing depth for bacteria; Supporting Information Fig. S14), the latter of which raises the doubts on whether there are significant differences on the understanding of microbial elevational range size using the two methods. (3) Furthermore, there was a significant $(P<0.001)$ relationship between the effortbased and coverage-based mean elevational range size for each taxon (Supporting Information Fig. S15). For the coverage-based mean elevational range size, we also observed variable elevational patterns in range size, ranging from significantly positive to negative relationships among taxonomic groups and elevational gradients (Supporting Information Fig. S16). Thus, either methods do not support a general elevational Rapoport's rule.

Second, quantification of the relationship between niche breadth and range size using non-independent data for calculating niche breadths may lead to spurious correlations and thus suspect conclusions. To avoid this problem, we related elevational range size for one elevational gradient with the mean niche breadth calculated using the data from the other mountains, taxon by taxon. One might envisage that our approach might still not result in independent measurements of niche breadth and species 
elevational range size because MAT declines monotonically with elevation at a lapse rate of around $6^{\circ} \mathrm{C}$ per $1000 \mathrm{~m}$ elevation globally. Regarding this concern in measuring temperature niche breadth, one has to create temperature gradients and place given species under different temperatures, and then estimate lethal limits of survival rates, critical temperature limits of 'ecological death' (Lutterschmidt and Hutchison, 1997), or long-term thermal distribution of individual species in the field. (1) One solution for estimating temperature niche breadths for each species is to measure them in laboratories. However, considering the hyperdiverse and mostly unculturable microbes (Locey and Lennon, 2016), or aquatic macroinvertebrates, it is not feasible to perform experiments in laboratories for high number of species nor there are existing experimental data sets available to be reviewed for species thermal performances as those for ectotherms (Sunday et al., 2011). Furthermore, experimentally determined temperature niche breadth possibly also inaccurately estimate the tolerance shown in situ because artificial laboratory conditions usually cannot consider biotic interactions and the variability in all natural environmental features. (2) Another solution is to use natural temperature gradients observed along latitude or elevations, the latter of which is the case here. Mountains, as natural laboratories, vary substantially in height, geological age, glaciation, landscape morphology, geochemical composition and magnitude of response to climate changes. Therefore, due to the variation in local environments and species dispersal/adaptation history across mountains, the linear relationship between elevation and temperature does not necessarily mean the invariability in realized species temperature niche breadth.

Our findings also provide two additional lines of evidence for the notion that the estimates of climatic niche breadth and species range size might be largely independent of each other. (1) Although species range size and temperature niche breadth were mostly significantly correlated, there was considerable variation in the correlations across mountains for each taxon, and there were also non-significant correlations observed for diatoms and macroinvertebrates (Supporting Information Figs S1 and S2). This suggests that their associations are not universal or spurious. (2) Mean annual temperature was not the most important dimension of niche breadth in explaining the mean elevational range size compared with climate variability and local variables (Fig. 3; Supporting Information Figs S5-S8). In sum, we thus think that our estimations for species range size and temperature niche breadth are as independent as possible, given the constraints of information available at this time.

Finally, we acknowledge that our findings on species range size and niche breadth are limited by sample size and the habitats we studied. For instance, only two European mountains and four mountains in China were examined for stream organisms. Further studies are encouraged to include more mountains or more samples per mountain with larger spatial scales for both independent, laboratory-based measurements of niche breadth and elevational range size for aquatic and terrestrial microbes. If as feasible as for macroorganisms, experimental manipulations of microbes in laboratories or field conditions for estimating niche breadth, independently of range size, would hopefully further support our current findings and enrich our knowledge of biodiversity theory in the study of biogeographical gradients in microbial range size.

\section{Conclusions}

For the first time, to the best of our knowledge, we present analyses of the elevational range size of microbes (bacteria and diatoms) and aquatic macroinvertebrates across large scales, which allows general conclusions on microbial range size to be made and allow us to compare these patterns with the corresponding findings in larger organisms. There was a consistent positive relationship between niche breadth and species elevational range size in all taxa, driven both by local environmental and climatic variables. Climatic variability was the dominant factor that explained the variation in mean elevational range size in microbes, whereas local environmental variables were more important for explaining the variation of mean range size in macroinvertebrates. For all taxonomic groups, elevational range size had significant positive relationships with diurnal temperature variability, and negative relationships with seasonal temperature variability, which gives insights into how thermal barriers for microbes constrain species range size via climate variability at these various temporal scales. We encourage observational studies and field experiments on mountainsides (Wang et al., 2016) for both aquatic and terrestrial ecosystems to carefully evaluate how the elevational range size of aquatic and terrestrial biota may differ in their response to short- and long-term climate variability.

\section{Materials and methods}

\section{Study area and field sampling}

We sampled six streams for bacteria and diatoms and four streams for macroinvertebrates along mountainsides in three regions (Wang et al., 2017): (1) one stream in the Balggesvarri Mountain region, (2) one stream in the Pyrenees Mountains in Spain, and (3) four streams in the Hengduan Mountain region in China. Macroinvertebrates were sampled only in the Hengduan mountain region.

Following the proposals of Wang et al. (2011; 2017), we sampled the complete elevational gradient when possible. Each study site was divided into 5 or 10 cross-sections. Twenty stones were selected randomly from riffle/run habitats, and biofilm was scraped off these stones. The subsamples were subsequently pooled into a composite sample at each 
site. The bacterial and water samples were frozen at $-18^{\circ} \mathrm{C}$. Four-kicknet samples of macroinvertebrates were stored in $70 \%$ ethanol.

\section{Physicochemical and biological analyses}

We logged latitude, longitude and elevation using a GPS unit. Shading (\% canopy cover), depth, current velocity, width, substratum particle size, water conductivity, $\mathrm{pH}$, temperature, cDOM, total nitrogen (TN) and TP were measured for each site (Wang et al., 2017).

Diatoms were identified to species level using microscopy, and bacteria were examined by high-throughput sequencing of $16 \mathrm{~S}$ rRNA (Wang et al., 2017). The diatoms and bacteria were rarefied at 500 cells and 10,000 sequences for further analyses respectively. Macroinvertebrates were identified to species level when possible using standard keys (Morse et al., 1994). Most Ephemeroptera, Plecoptera, Trichoptera, Coleoptera, Diptera, Hemiptera, Lepidoptera and Odonata were identified to species level (Wang et al., 2011). The identification level of all other taxonomic groups varied from species to family. Oligochaeta were identified to class level only.

\section{Climatic variables}

We used climatic variables from the WorldClim database (Hijmans et al., 2005). The variables included were MAT, MDR, TS and TAR. The selected variables are expected to indicate major temperature patterns and variation (Chan et al., 2016). According to the WorldClim database, climatic variation among seasons increases with elevation, which is an important prerequisite for the ERR. We expected that such large temporal variation in climatic factors may also be reflected in larger temporal variations in water chemistry toward higher elevations (e.g., through more variable hydrological conditions), although this could not be tested due to the lack of temporal data on water chemistry.

\section{Data analyses}

We considered range size at two different scales: the species level and the community level. (1) For the species level analyses, the species elevational range size was estimated for each stream as the maximum range extent, assuming that a species was present between its highest and lowest detected elevations along a single montane gradient (Gaston, 2003). When a species was detected only at one site, the species elevational range size was assigned as the half of the shortest elevational differences among sites in each stream (Cardelus et al., 2006).

To evaluate the phylogenetic signal of species elevational range size across a range of phylogenetic depths, we used Mantel correlograms with 999 randomizations for significance tests with the function 'mantel.correlog' in the Vegan v2.0-2 R package (http://vegan.r-forge.r-project.org) (Wang et al., 2013). We partitioned phylogenetic distances into classes (i.e., evolutionary time steps; here, 0.02 units), and within each distance class we determined the correlation coefficient relating between-OTU phylogenetic distances to species elevational range size differences (Wang et al., 2013). Because phylogenetic information was unavailable for diatoms and macroinvertebrates, we tested phylogenetic signal only in bacteria.

The measured environmental variables were grouped into five niche categories: (a) stream morphology (stream width, shading, substratum size, depth and current velocity), (b) chemistry ( $\mathrm{pH}$ and conductivity), (c) nutrients (TP and cDOM), (d) MAT, and (e) climatic variation (MDR, TS and TAR). We calculated species niche breadth as the variance of the standardized environmental variables (mean $=0 ; S D=1$ ) of the samples in which species occurred or the sum of the variances of the standardized variables of each niche category. To use trait data that were as independent as possible for the niche breadth-range size analyses for each stream, we analysed the NBRS hypothesis for each stream in a 'leave one out' bootstrap design using the niche breadth data from the other five streams, for the corresponding taxa. We examined the Pearson correlation coefficient $r$ for NBRS, and then calculated the common effect size as Fisher's $z$, which is the standard effect size measure used for correlational data (Borenstein et al., 2009). In total, we extracted 80 effect sizes from five niche categories and three taxonomic groups for six streams.

We then ran random-effects models for each niche category or taxonomic group. The null hypothesis for each analysis was that the mean effect size was zero. The effect sizes were calculated in such a way that a positive value indicated that species with a broader niche occupied a greater elevational range. Heterogeneity was estimated using the $f$ statistic (Higgins and Thompson, 2002).

To examine the influence of niche category on support for the NBRS hypothesis, we ran separate mixed-effects models for each taxonomic group using effect sizes as the response variables and the niche categories as explanatory factors. To further examine the influence of taxonomic group on NBRS, we ran separate mixed-effects models for each niche category using effect sizes as response variables and taxonomic groups as explanatory factors. Both random-effects and mixed-effects models were performed using the Metafor $\mathrm{R}$ package (Viechtbauer, 2010).

(2) For the community level analyses, the mean elevational range size for the community was estimated using two methods: the Stevens (Stevens, 1992) and the McCain (McCain and Bracy, 2013) methods. The Stevens method quantifies the average range size of all the species in a community. According to the ERR, we expect a positive association between average range size and elevation. The McCain method considers whether small-ranged species are predominantly found at the lowest elevations and decrease in density with increasing elevation (McCain and Bracy, 2013). The smallest-range-size quartile is defined as the species of less than a quarter of maximum elevational range between sites. In this case, we expected positive trends as the smallest ranges would be expected to predominate at the lowest elevations while the frequency of the smallest ranges decreases with increasing elevation (as predicted by the ERR) (McCain and Bracy, 2013). The relationships between elevational range size and elevation, between species richness and range size, and between range size and environmental variables were explored with linear and quadratic regression models. The best models were selected based on AIC (Yamaoka et al., 1978).

The relationships between mean elevational range size and potential explanatory variables were further analysed using BRT for the whole data sets covering the six streams or for the 
data sets only considering the Chinese streams. BRT is an ensemble method for fitting statistical models, different from most conventional techniques that aim to fit a single parsimonious model (Elith et al., 2008). BRT is based on the combination of the strengths of two algorithms: regression trees (models that relate a response to their predictors by recursive binary splits) and boosting (an adaptive method for combining many simple models to give improved predictive performance). The final BRT model can be understood as an additive regression model in which individual terms are simple trees, fitted in a forward, stagewise fashion (Elith et al., 2008). We considered the following explanatory variables: streamwater temperature, $\mathrm{pH}$, conductivity, TP, cDOM, stream width, depth, current velocity, substratum size, stream shading and the four climatic variables. All the explanatory environmental variables were standardized with a mean $=0$ and an $S D=1$. As all relationships between explanatory variables were lower than Spearman's $\rho^{2}=0.65$, we kept all variables in the models. Given the relatively low sample size, we kept the size of trees, and the consequent interactions' order, low (tree complexity parameter $=2$ ), and chose a low shrinkage parameter (learning rate parameter $=0.002$ ), thereby controlling the contribution of each individual tree to the final model. We produced an optimal number of trees of at least 1000, using cross-validation (Elith et al., 2008). The importance of a predictor variable was determined by its frequency of selection (for splitting) weighted by a measure of model improvement given each split and averaged across all trees (contributions were scaled to sum to 100). All BRT results (variable importance and predictions) were averaged across the m-imputed datasets. BRT analyses were implemented with the $\mathrm{gbm} \mathrm{R}$ package (V. 2.1, https://cran.r-project.org/web/packages/gbm).

\section{Data accessibility}

The sequences were deposited in MG-RAST database under the accession number 17666 .

\section{Acknowledgements}

We are grateful to CY Zhang, Y Zhang, E. Casamayor, X Triado-Margarit, S. Meier, C Plum, J Eskelinen, BX Wang, Q Wang, YL Zhang, JZ Zhou, J Shen, and XM Tang for field sampling, lab analyses or data providing, to ZJ Wang and ZS An for fund notice. We sincerely thank Robert Colwell for dedicated proofreading and constructive comments, which greatly improved the manuscript. JW was supported by Key Research Program of Frontier Sciences, Chinese Academy of Sciences (QYZDB-SSW-DQC043), The Program of Global Change and Mitigation (2017YFA0605200, 2016YFA0600502), NSFC grants $(41571058,41273088)$ and The National Geographic Air and Water Conservation Fund (GEFC12-14). JS and JW were supported by Emil Aaltonen Foundation. The authors declare no conflict of interest.

\section{Author contributions}

JS provided diatom data. JW performed field experiments, provided the other data and performed statistical analyses. Both authors wrote the manuscript.

\section{References}

Angert, A.L., Crozier, L.G., Rissler, L.J., Gilman, S.E., Tewksbury, J.J., and Chunco, A.J. (2011) Do species' traits predict recent shifts at expanding range edges?. Ecol Lett 14: 677-689.

Arita, H., Christen, J., Rodríguez, P., and Soberón, J. (2008) Species diversity and distribution in presence-absence matrices: mathematical relationships and biological implications. Am Nat 172: 519-532.

Azovsky, A., and Mazei, Y. (2013) Do microbes have macroecology? Large-scale patterns in the diversity and distribution of marine benthic ciliates. Glob Ecol Biogeogr 22: 163-172.

Barberán, A., Ramirez, K.S., Leff, J.W., Bradford, M.A., Wall, D.H., and Fierer, N. (2014) Why are some microbes more ubiquitous than others? Predicting the habitat breadth of soil bacteria. Ecol Lett 17: 794-802.

Beketov, M.A. (2009) The Rapoport effect is detected in a river system and is based on nested organization. Glob Ecol Biogeogr 18: 498-506.

Bhatt, J.P., Manish, K., and Pandit, M.K. (2012) Elevational gradients in fish diversity in the Himalaya: water discharge is the key driver of distribution patterns. PLoS One 7: e46237.

Borenstein, M., Hedges, L.V., Higgins, J., and Rothstein, H.R. (2009) Introduction to Meta-Analysis. New York: Wiley Online Library.

Brown, J.H. (1984) On the relationship between abundance and distribution of species. Am Nat 124: 255-279.

Brown, J.H., Stevens, G.C., and Kaufman, D.M. (1996) The geographic range: size, shape, boundaries, and internal structure. Annu Rev Ecol Syst 597-623.

Cardelus, C.L., Colwell, R.K., and Watkins, J.E. (2006) Vascular epiphyte distribution patterns: explaining the midelevation richness peak. J Ecol 94: 144-156.

Chan, W.-P., Chen, I.-C., Colwell, R.K., Liu, W.-C., Huang, Cy., and Shen, S.-F. (2016) Seasonal and daily climate variation have opposite effects on species elevational range size. Science 351: 1437-1439.

Chao, A., and Jost, L. (2012) Coverage-based rarefaction and extrapolation: standardizing samples by completeness rather than size. Ecology 93: 2533-2547.

Chen, I.-C., Hill, J.K., Ohlemüller, R., Roy, D.B., and Thomas, C.D. (2011) Rapid range shifts of species associated with high levels of climate warming. Science 333: 1024-1026.

Colwell, R.K. (2011) Biogeographical gradient theory. In The Theory of Ecology. Scheiner, S.M., and Willig, M.R. (eds). Chicago: University of Chicago Press, pp. 309-330.

Colwell, R.K., and Hurtt, G.C. (1994) Nonbiological gradients in species richness and a spurious Rapoport effect. Am Nat 144: 570-595.

Colwell, R.K., and Lees, D.C. (2000) The mid-domain effect: geometric constraints on the geography of species richness. Trends Ecol Evol 15: 70-76.

Dudgeon, D., and Wu, K.K. (1999) Leaf litter in a tropical stream: food or substrate for macroinvertebrates? Arch Hydrobiol 146: 65-82.

Eckert, C., Samis, K., and Lougheed, S. (2008) Genetic variation across species' geographical ranges: the central-marginal hypothesis and beyond. Mol Ecol 17: 1170-1188.

Elith, J., Leathwick, J.R., and Hastie, T. (2008) A working guide to boosted regression trees. J Anim Ecol 77: 802-813. 
Fattorini, S. (2014) Disentangling the effects of available area, mid-domain constraints, and species environmental tolerance on the altitudinal distribution of tenebrionid beetles in a Mediterranean area. Biodivers Conserv 23: 2545-2560.

Fierer, N., Strickland, M.S., Liptzin, D., Bradford, M.A., and Cleveland, C.C. (2009) Global patterns in belowground communities. Ecol Lett 12: 1238-1249.

Fu, C., Wu, J., Wang, X., Lei, G., and Chen, J. (2004) Patterns of diversity, altitudinal range and body size among freshwater fishes in the Yangtze River basin, China. Glob Ecol Biogeogr 13: 543-552.

Gaston, K.J. (2003) The Structure and Dynamics of Geographic Ranges. Oxford: Oxford University Press.

Gaston, K.J., and Blackburn, T.M. (2000) Pattern and Process in Macroecology. New York: Wiley.

Graves, G.R., and Rahbek, C. (2005) Source pool geometry and the assembly of continental avifaunas. Proc Natl Acad Sci USA 102: 7871-7876.

Hanski, I. (1982) Dynamics of regional distribution: the core and satellite species hypothesis. Oikos 210-221.

Hanson, C.A., Fuhrman, J.A., Horner-Devine, M.C., and Martiny, J.B.H. (2012) Beyond biogeographic patterns: processes shaping the microbial landscape. Nat Rev Microbiol 10: 497-506.

Higgins, J., and Thompson, S.G. (2002) Quantifying heterogeneity in a meta-analysis. Stat Med 21: 1539-1558.

Hijmans, R.J., Cameron, S.E., Parra, J.L., Jones, P.G., and Jarvis, A. (2005) Very high resolution interpolated climate surfaces for global land areas. Int J Climatol 25: 1965-1978.

Hsieh, T.C., Ma, K.H., and Chao, A. (2016) iNEXT: an R package for rarefaction and extrapolation of species diversity (Hill numbers). Methods Ecol Evol 7: 1451-1456.

Janzen, D.H. (1967) Why mountain passes are higher in the tropics. Am Nat 101: 233-249.

Klopfer, P.H., and MacArthur, R.H. (1960) Niche size and faunal diversity. Am Nat 94: 293-300.

Kraft, N.J.B., Comita, L.S., Chase, J.M., Sanders, N.J., Swenson, N.G., Crist, T.O., et al. (2011) Disentangling the drivers of $\beta$ diversity along latitudinal and elevational gradients. Science 333: 1755-1758.

Krosby, M., Wilsey, C.B., McGuire, J.L., Duggan, J.M., Nogeire, T.M., Heinrichs, J.A., et al. (2015) Climate-induced range overlap among closely related species. Nat Clim Change 5: 883-886.

Laube, I., Graham, C.H., and Böhning-Gaese, K. (2013) Intrageneric species richness and dispersal ability interact to determine geographic ranges of birds. Glob Ecol Biogeogr 22: 223-232.

Legendre, P. (2014) Interpreting the replacement and richness difference components of beta diversity. Glob Ecol Biogeogr 23: 1324-1334.

Legendre, P., and De Cáceres, M. (2013) Beta diversity as the variance of community data: dissimilarity coefficients and partitioning. Ecol Lett 16: 951-963.

Liu, Y., Priscu, J.C., Yao, T., Vick-Majors, T.J., Xu, B., Jiao, N., et al. (2016) Bacterial responses to environmental change on the Tibetan Plateau over the past half century. Environ Microbiol 18: 1930-1941.

Locey, K.J., and Lennon, J.T. (2016) Scaling laws predict global microbial diversity. Proc Natl Acad Sci USA 113: 5970-5975.
Lutterschmidt, W.I., and Hutchison, V.H. (1997) The critical thermal maximum: history and critique. Can $J$ Zool 75: 1561-1574.

MacArthur, R.H. (1968) The theory of the niche. Popul Biol Evol 159: 176.

Martiny, J.B.H., Bohannan, B.J.M., Brown, J.H., Colwell, R.K., Fuhrman, J.A., Green, J.L., et al. (2006) Microbial biogeography: Putting microorganisms on the map. Nat Rev Microbiol 4: 102-112.

McCain, C.M. (2009) Vertebrate range sizes indicate that mountains may be 'higher' in the tropics. Ecol Lett 12: 550560.

McCain, C.M., and Colwell, R.K. (2011) Assessing the threat to montane biodiversity from discordant shifts in temperature and precipitation in a changing climate. Ecol Lett 14: 1236-1245.

McCain, C.M., and Bracy, K.K. (2013) Elevational Rapoport's rule is not pervasive on mountains. Glob Ecol Biogeogr 22: 750-759.

Mellin, C., Mouillot, D., Kulbicki, M., McClanahan, T.R., Vigliola, L., Bradshaw, C.J.A., et al. (2016) Humans and seasonal climate variability threaten large-bodied coral reef fish with small ranges. Nat Commun 7: 10491.

Miyamoto, Y., Nakano, T., Hattori, M., and Nara, K. (2014) The mid-domain effect in ectomycorrhizal fungi: range overlap along an elevation gradient on Mount Fuji, Japan. ISME J 8: 1739-1746.

Morse, J., Yang, L., and Tian, L. (1994) Aquatic Insects of China Useful for Monitoring Water Quality. Nanjing: Hohai University Press.

Morueta-Holme, N., Enquist, B.J., McGill, B.J., Boyle, B., Jørgensen, P.M., Ott, J.E., et al. (2013) Habitat area and climate stability determine geographical variation in plant species range sizes. Ecol Lett 16: 1446-1454.

Mykrä, H., Heino, J., and Muotka, T. (2007) Scale-related patterns in the spatial and environmental components of stream macroinvertebrate assemblage variation. Glob Ecol Biogeogr 16: 149-159.

Pajunen, V., Luoto, M., and Soininen, J. (2016) Climate is an important driver for stream diatom distributions. Glob Ecol Biogeogr 25: 198-206.

Papacostas, K.J., and Freestone, A.L. (2016) Latitudinal gradient in niche breadth of brachyuran crabs. Glob Ecol Biogeogr 25: 207-217.

Payne, N.L., and Smith, J.A. (2017) An alternative explanation for global trends in thermal tolerance. Ecol Lett 20: 70-77.

Rohner, P.T., Bächli, G., Pollini Paltrinieri, L., Duelli, P., Obrist, M.K., Jochmann, R., and Blanckenhorn, W.U. (2015) Distribution, diversity gradients and Rapoport's elevational rule in the black scavenger flies of the Swiss Alps (Diptera: Sepsidae). Insect Conserv Divers 8: 367-376.

Sheldon, K.S., Leaché, A.D., and Cruz, F.B. (2015) The influence of temperature seasonality on elevational range size across latitude: a test using Liolaemus lizards. Glob Ecol Biogeogr 24: 632-641.

Slatyer, R.A., Hirst, M., and Sexton, J.P. (2013) Niche breadth predicts geographical range size: a general ecological pattern. Ecol Lett 16: 1104-1114.

Soberón, J., and Ceballos, G. (2011) Species richness and range size of the terrestrial mammals of the world: biological signal within mathematical constraints. PLoS One 6: e19359. 
Soininen, J. (2007) Environmental and spatial control of freshwater diatoms - A review. Diatom Res 22: 473-490.

Soininen, J., Jamoneau, A., Rosebery, J., and Passy, S.I. (2016) Global patterns and drivers of species and trait composition in diatoms. Global Ecol Biogeogr 25: 940-950.

Spicer, J., and Gaston, K. (2009) Physiological Diversity: Ecological Implications. New York: John Wiley \& Sons.

Stevens, G.C. (1989) The latitudinal gradient in geographical range: how so many species coexist in the tropics. Am Nat 240-256.

Stevens, G. (1992) The elevational gradient in altitudinal range: an extension of Rapoport's latitudinal rule to altitude. Am Nat 140: 893-911.

Sunday, J.M., Bates, A.E., and Dulvy, N.K. (2011) Global analysis of thermal tolerance and latitude in ectotherms. Proc Biol Sci 278: 1823-1830.

Teittinen, A., Kallajoki, L., Meier, S., Stigzelius, T., and Soininen, J. (2016) The roles of elevation and local environmental factors as drivers of diatom diversity in subarctic streams. Freshw Biol 61: 1509.

Tettelin, H., Riley, D., Cattuto, C., and Medini, D. (2008) Comparative genomics: the bacterial pan-genome. Curr Opin Microbiol 11: 472-477.

Thuiller, W., Lavorel, S., Araújo, M.B., Sykes, M.T., and Prentice, I.C. (2005) Climate change threats to plant diversity in Europe. Proc Natl Acad Sci USA 102: 8245-8250.

Trakimas, G., Whittaker, R.J., and Borregaard, M.K. (2016) Do biological traits drive geographical patterns in European amphibians? Glob Ecol Biogeogr 25: 1228-1238.

Viechtbauer, W. (2010) Conducting meta-analyses in R with the metafor package. J Stat Softw 36: 1-48.

Wang, J., Soininen, J., Zhang, Y., Wang, B., Yang, X., and Shen, J. (2011) Contrasting patterns in elevational diversity between microorganisms and macroorganisms. J Biogeogr 38: 595-603.

Wang, J., Shen, J., Wu, Y., Tu, C., Soininen, J., Stegen, J.C., et al. (2013) Phylogenetic beta diversity in bacterial assemblages across ecosystems: deterministic versus stochastic processes. Isme J 7: 1310-1321.

Wang, J., Pan, F., Soininen, J., Heino, J., and Shen, J. (2016) Nutrient enrichment modifies temperature-biodiversity relationships in large-scale field experiments. Nat Commun 7: 13960.

Wang, J., Meier, S., Soininen, J., Casamayor, E., Tang, X., Yang, X., et al. (2017) Regional and global elevational patterns of microbial species richness and evenness. Ecography 40: 393-402.

Wiens, J.J. (2016) Climate-related local extinctions are already widespread among plant and animal species. PLoS Biol 14: e2001104.

Yamaoka, K., Nakagawa, T., and Uno, T. (1978) Application of Akaike's information criterion (AIC) in the evaluation of linear pharmacokinetic equations. J Pharmacokinet Biopharm 6: $165-175$.

\section{Supporting information}

Additional Supporting Information may be found in the online version of this article at the publisher's web-site:

Table S1. Results of mixed-effects meta-analysis examining the influence of niche category on the relationship between niche breadth and elevational range. $Q_{\mathrm{M}}$ and the associated
$P$-value provide a test for the effect of niche category on the mean effect size, while $Q_{E}$ provides a test of residual heterogeneity, estimated by $\tau^{2}$. Morphology breadth was used as the reference level.

Table S2. Results of mixed-effects meta-analysis examining the influence of taxonomic group on the relationship between niche breadth and elevational range. $Q_{\mathrm{M}}$ and the associated $P$-value provide a test for the effect of taxonomic groups on the mean effect size, while $Q_{E}$ provides a test of residual heterogeneity, estimated by $\tau^{2}$. Bacteria was used as the reference level.

Fig. S1. The relationships between mean annual temperature (MAT) breadth and species elevational range size. Th relationship for each panel was fitted by a linear model, the significance of which $(P \leq 0.05, F$-test) are shown with red solid lines. Gray lines indicate non-significant relationships of a linear model $(P>0.05, F$-test). The blue dotted lines indicate the locally weighted scatterplot smoothing regressions.

Fig. S2. The Pearson correlation coefficient $r$ of the relationships between niche breadth and species elevational range size. The niche breadth categories are stream morphology, streamwater chemistry, streamwater nutrients, mean annual temperature (MAT) and climatic variation. Grey dots indicate the non-significant Pearson correlations $(P>0.05)$. The details of the relationships between niche breadth of MAT and species elevational range size are shown in Supporting Information Fig. S1.

Fig. S3. The relationships between the frequency of smallest-ranged species and elevation. The smallest-ranges species were defined as the species of less than a quarter of the maximum of elevational range among sites according to McCain's method ${ }^{1}$. The relationship for each panel was fitted by linear and quadratic models, the significances of which $(P \leq 0.05, F$-test) are shown with blue and red lines. The better model was selected based on the lower value of Akaike's Information Criterion, and is shown as a solid line. Adjusted $R^{2}$ values are shown for significant $(P \leq 0.05, F-$ test) linear (blue) and quadratic (red) regressions. Nonsignificant $(P>0.05, F$-test $)$ relationships are shown by grey dotted lines.

Fig. S4. The relationships between community mean elevational range size and species richness. Mean elevational range size is the mean value of species elevational range for all species in the community based on Stevens' method. Species richness was calculated based on equal sampling, and did not consider equal sample coverage. The relationship for each panel was fitted by linear and quadratic models, the significance of which $(P \leq 0.05, F$-test) is shown for blue lines. Adjusted $r^{2}$ values are shown for significant $(P \leq 0.05)$ linear regressions. Species richness in each panel was standardized (mean $=0 ; S D=1$ ) for better visualization.

Fig. S5. The relationships between community mean elevation range size and environmental variables for bacteria. Mean elevational range size is the mean value of species elevation range size for all species in the community based on Stevens' method. The relationship for each panel was fitted by linear and quadratic models, the significances of which $(P \leq 0.05, F$-test) are shown for blue and red lines. The better model was selected based on the lower value of 
Akaike's Information Criterion, and is shown as solid line. Adjusted $R^{2}$ values are shown for significant $(P<0.05, F$ test) linear (blue) and quadratic (red) regressions. Nonsignificant $(P>0.05, F$-test) relationship was shown in grey dotted line. All of the environmental variables were standardized (mean $=0 ; S D=1$ ) for better visualization.

Fig. S6. The relationships between community mean elevation range size and environmental variables for diatoms. The model fittings are the same in Supporting Information Fig. S5.

Fig. S7. The relationships between community mean elevation range size and environmental variables for macroinvertebrates. The model fittings are the same in Supporting Information Fig. S5.

Fig. S8. Partial dependence plots for all variables included in the Boosted Regression Trees ${ }^{2}$ model of bacterial elevational range size. The $y$-axes represent the marginal effect of the respective explanatory variable on the predicted species richness (centred to zero mean). The relative contribution (\%) of each variable is shown in parentheses on the $x$-axes. The relative contributions are visualized in Fig. 3. The solid line is the BRT-modelled response curve to the most important variables. The dashed line is the locally weighted scatterplot smoothing (LOESS) regression (span 0.25) fitted to the response curve. TP: total phosphorus. Shading: riparian shading (\%). Substratum: median of the substratum particle size. Velocity: current velocity. Depth: streamwater depth. cDOM: chromophoric dissolved organic matter. Temperature: streamwater temperature. MAT: mean annual temperature. MDR: mean diurnal range. TS: temperature seasonality. TAR: temperature annual range.

Fig. S9. Partial dependence plots for all variables included in the Boosted Regression Trees model of diatom elevational range size. The other figure legend is the same in Supporting Information Fig. S8.

Fig. S10. The environmental factors related to the community elevational range size, identified with Boosted Regression Trees (BRT). The results were for bacteria (A) and diatoms (B) from the four streams in China. The values of the relative contribution (\%) of each variable for each biodiversity metric are shown. TP: total phosphorus. Shading: riparian shading (\%). Substratum: median of the substratum particle size. Velocity: current velocity. Depth: streamwater depth. cDOM: chromophoric dissolved organic matter. Temperature: streamwater temperature. MAT: mean annual temperature. MDR: mean diurnal range. TS: temperature seasonality. TAR: temperature annual range.

Fig. S11. Partial dependence plots for all variables included in the Boosted Regression Trees model of macroinvertebrate elevational range size. The other figure legend is the same in Supporting Information Fig. S8.

Fig. S12. The relationships between community mean elevational range size and sample coverage-based rarefied species richness. Mean elevational range size is the mean values of species elevational range for all species in the community based on Stevens' method. The species richness was calculated with iNEXT ${ }^{3}$ based on the lowest species coverages of $0.921,0.951$ and 0.830 for bacteria, diatoms, and macroinvertebrates respectively. iNEXT uses the occurrence of singletons and doubletons to estimate completeness of all the samples, identifying the sample with the lowest species coverage ${ }^{4}$. The relationship for each panel was fitted by linear and quadratic models, the significances of which $(P \leq 0.05, F$-test) are shown by blue lines. Adjusted $r^{2}$ values are shown for significant $(P \leq 0.05)$ linear regressions. Species richness in each panel was standardized (mean $=0 ; S D=1$ ) for better visualization.

Fig. S13. The relationships between effort-based (SE) and coverage-based (SC) species richness. The effort-based species richness was calculated based on the raw data obtained with even field sampling effort, while the coveragebased species richness was calculated based on the lowest sample coverage for each taxa. It should be noted that the effort-based species richness for bacteria and diatoms was calculated based on the species $X$ site matrix without any further rarefaction. The blue solid lines indicate the loess regressions. These results show the visually high correlations between the two estimates of species richness, and the correlation is extremely strong $(P<0.001)$ if the estimates are calculated based on exactly comparable observation efforts among samples (i.e., the same sequencing depth for bacteria, as shown in Supporting Information Fig. S14).

Fig. S14. The relationships between effort-based (SE) and coverage-based (SC) species richness for bacteria. The effort-based species richness was calculated based on the species $X$ site matrix with the rarefaction of 10,000 sequences, while the coverage-based species richness was calculated based on the rarefied matrix of the lowest species coverage. The loess regression is shown with blue solid line.

Fig. S15. The relationships between the mean elevational range sizes calculated with two data matrices: sampling effort-based (SE) and sample coverage-based (SC). The elevational range sizes were calculated according to Stevens' method based on a species $X$ site matrix of even sampling efforts (that is, effort-based) and an alternative species $X$ site matrix, rarefied according to the lowest species coverage for each taxon (that is, coverage-based). The lowest species coverages were calculated with iNEXT ${ }^{3}$. The relationships between $\mathrm{SE}$ and $\mathrm{SC}$ range sizes were fitted by highly significant $(P<0.001)$ linear models, indicated with blue solid lines.

Fig. S16. The relationships between the mean community elevational range size and elevation. The elevational range sizes were calculated according to Steven's method based on an alternative species $X$ site matrix, rarefied according to the lowest species coverage for each taxa. The lowest species coverages (Supporting Information Fig. S12) were calculated with iNEXT ${ }^{3}$. The relationship for each panel was fitted by linear and quadratic models, the significances of which $(P \leq 0.05, F$-test) are shown with blue and red lines. The better model was selected based on the lower value of Akaike's Information Criterion, and is shown as solid line. Adjusted $R^{2}$ values are shown for significant $(P \leq 0.05, F$ test) linear (blue) and quadratic (red) regressions. Nonsignificant $(P>0.05, F$-test) relationship is shown in a grey dotted line. 